\title{
Wavelet Analysis of the Wind Velocity and Temperature Variability in the Amazon Forest
}

\author{
Maurício José Alves Bolzan and Paulo Cesar Vieira \\ Laboratório de Física Atmosférica e Solar, Instituto de Pesquisa e Desenvolvimento, \\ Universidade do Vale do Paraíba, UNIVAP, Av. Shishima Hifumi, \\ n. 2911, 12244-000, Urbanova, São José dos Campos, SP, Brazil
}

Received on 14 June, 2006

\begin{abstract}
We studied the turbulent interactions among vertical wind velocity and temperature time-series measured in the Amazonian forest, during the wet season campaign of Large Biophere-Atmosphere Experiment in Amazonia (LBA) in 1999. The approach is based on the estimation of the correlation coefficient between the different scales in turbulent fields and Cross Wavelet Power (XWP). The results suggest that the correlations among scales of the vertical wind velocity are due to the Coherent Structures (CS), a large scale signature in the thermal profile. These coherent structures, kind of ramps, promoted an increase in the interaction among both variables, vertical wind velocity and temperature, and also depends on the atmospheric stability conditions. Furthermore, these coherent structures may explain the higher values of the correlation coefficient found in the large scales during the diurnal period compared with the nocturnal period, for the vertical wind velocity.
\end{abstract}

Keywords: Intermittence; Temperature; Wind; Turbulence; Amazonia; Statistical Analysis

\section{INTRODUCTION}

The study of the interactions among large scales structures in the turbulent flows has been of growing interest ([1], [2], [3], [4], [5], [6] and references there in). This subject is of very practical interest, mainly in the study of the dispersion properties of the passive scalars in Atmospheric BoudaryLayer (ABL). In this aspect, Warhaft [7] has done an excellent review of the main properties of scalar as temperature, showing important differences between temperature and velocity turbulent fields found in numerical simulations and experimental studies.

In this paper, we report our results on large scales interactions in turbulent fields addressing both the vertical wind velocity and temperature measured over the Amazon forest. We used an approach based on the Katul's methodology [8] to characterize the correlation level among large scales in the vertical wind velocity. If these correlations exist, due to the velocity field eddies, it would be possible to associate them with a passive scalar large scale variability as the forest environmental temperature. Robinson [9] has defined the eddies as Coherent Structures (CS) in turbulent regime where the variables have high self-correlation level being able to show a correspondent phase space signature [10]. The study of this subject is very important in several areas e.g. space science ([11], [12], [6]) and meteorology ([3]).

The CS approach ([3],[9]) support the idea that there are interactions among structures of different scales and between velocity and temperature variables. In particular for temperature, it is important to point out that, the shear flows conditions play an important role for the scalar spectrum ([13], [14], [7]). This aspect needs to be considered for roughness flow surface as the Amazonia forest. Thus, the objective of this work is to investigate possible influence of the thermal CS on the large scales eddies of vertical wind velocity, measured in the Amazonian forest, in both atmospheric conditions, diurnal and nocturnal periods.

\section{METHODOLOGY}

To study the interactions between different scales, we used the Katul's methodology [8], which is based on the fact that any time series value $x$, can be characterized by the difference of velocity $\Delta w=w(x+r)-w(x)$. Thus, a measure of interaction between two different flow scales is given by the correlation coefficient $\rho_{w, \Delta w}$, defined as:

$\rho_{w, \Delta w}(r)=\frac{<(w(x)-<w(x)>)(\Delta w(x, r)-<\Delta w(x, r)>)>}{\sigma_{w} \sigma_{\Delta w}}$,

were $\sigma_{y}$ is the standard deviation of the variable $y$ of the flow, and given by $\sigma_{y}=<(y-<y>)^{2}>^{1 / 2}$, with $<$. $>$ denoting the mean value of the enclosed quantity and $r$ is the increment in logarithimic space (it is similar to equation used by Osborne [15]). To obtain the term $\Delta w(x, r)-<\Delta w(x, r)>$ from equation (1), we used the Haar wavelet transform to decompose the time-series in $r$ dyadic scales (frequencies) [16]. However, to understand better the subject of wavelet decomposition in the context of this work, it is important to give a brief theoretical introduction.

The wavelet word indicate a set of functions with forms of little waves created by dilations, $\Psi(t)=\Psi(2 t)$, and translations, $\Psi(t)=\Psi(t+1)$, from a simple generator function, $\Psi(t)$, called mother-wavelet. Mathematically, the Wavelet Transform (WT) is defined by:

$$
\left(W_{\Psi} f\right)(a, b)=\frac{1}{a^{1 / 2}} \int_{\mathfrak{R}} f(t) \Psi^{*}\left(\frac{t-b}{a}\right) d t
$$

where the temporal function $f(t)$ is any time-series and $\Psi^{*}$ is the complex conjugate of the wavelet function in scale $a>1$ and position $b$.

In wavelet theory, there exists two types of wavelet functions: discrete and continuous. The discrete wavelets are able to decompose the time-series in all possible dyadic scales, i.e., 
the time series have a number of samples that is in power of two (e.g., $8192=2^{13}$ ). The Haar wavelet uses a rectangular window to sample the time series. The first pass over the time series uses a window width of two. The window width is doubled at each step until the window encompasses the entire time series. Thus, each pass over the time series generates a new time series. At the end, we will have the 13 new time series as shown in Fig.(1), where we have the 13 scales of one vertical velocity time-series (in order to improve the variability pattern, the last six time series, by summation, are sample as one). Furthermore, we used the Morlet wavelet transform, that is a continuous function, to evaluate the cross wavelet of two time-series $x_{n}$ and $y_{n}$. The cross wavelet power (XWP) is defined as in ([17], [18]) following:

$$
\left|W_{x y}\right|=a b s\left(\frac{\left|W_{x} W_{y}^{*}\right|}{\sigma_{x} \sigma_{y}}\right)
$$

where $*$ denotes complex conjugation, and $\sigma_{x}$ and $\sigma_{y}$ are the respective standard deviations, and abs symbol denote the absolute values from $W_{x y}$. For more information about this subject see Bolzan [19] and references there in.

The XWP give us information about the correlation between two variables in time and period. However, to study the main periods where the correlation is higher, we used the procedure called the Global Wavelet Spectrum (GWS) [20]. This mathematical tool is given by the following equation:

$$
G W S=\left(\frac{\sigma_{w} \sigma_{T}}{\sigma_{w}+\sigma_{T}}\right) \int\left|W_{w T}(a, b)\right|^{2} d b,
$$

where $\sigma_{w}$ e $\sigma_{T}$ are variance of the vertical wind velocity and temperature, respectively. Equation (4) is similar to the equation proposed by Le and Wang [21]. However, the difference here is that the variances $\left(\sigma_{w}\right.$ and $\left.\sigma_{T}\right)$ are considered explicitly. Furthermore, we performed the Fourier co-spectrum of $w$ and $T$ to compare the results between the Fourier co-spectrum and GWS.

\section{DATA AND EXPERIMENTAL SITE}

The Large Scale Biosphere-Atmosphere Experiment in Amazonia (LBA) is an international research initiative led by Brazil through the National Institute of Space Research (INPE). The LBA is designed to create new knowledge needed to understand the climatological, ecological, biogeochemical, hydrological processes and environmental physics of the Amazonia region (hereafter referred as Amazonia), the impact of land use change on these functions, and the interactions between the Amazonia and Earth system. In this work, we used the data obtained during an intensive micrometeorological campaign, in the context of wet-season LBA project. The experiment was carried out during the months of January to March 1999. Measurements were made simultaneously at different heights in a micrometeorological tower located in the Biological Reserve of Jaru (Jaru'REBIO: $10^{\circ} 04^{\prime} S 61^{\circ} 56^{\prime} W$ ),
Brazil. The heights are: Upper Level (66 meters - above the forest canopy); Medium Level (45 meters - at the top of canopy); and Lower Level (21 meters - below the canopy). The fast response wind speed measurements, sampled at 60 $\mathrm{Hz}$, were made using three-dimensional sonic anemometers (Campbell Scientific Inc, model CSAT-3-L60). The temperatures were measured using sonic thermometers (Campbell Scientific Inc, model CA27) [22].

During the experiment, the state of the atmosphere was characterized by the existence of strong convective activity in the diurnal period with isolated rain events and also, by the influence of the Convergence Zone of Southern Atlantic which was active above the Brazilian State of Rondônia (SouthWestern Amazonia) [22]. The experimental site was characterized by the existence of reasonable fetching conditions. The area where the tower was built is surrounded by the Amazon Forest in a radius of at least $800 \mathrm{~m}$ around the tower. The topography of the area is not totally homogeneous, since at the southern and eastern sides of the tower there are small hills with heights of some tens of meter More information on the variability pattern of the main micrometeorological variables during the period of the experiment is presented by Silva Dias [22].

We used seven time-series of the vertical wind velocity component (w) and temperature (T), measured at upper level, for both diurnal and nocturnal periods that are under unstable and stable conditions, respectively. Further, we checked the vertical velocity power spectrum, which displayed a sizeable scaling range with a slope of approximately $-5 / 3$ in the inertial subrange. Fig.(2) shows the Power Spectrum Density (PSD) for $\mathrm{w}$ and $\mathrm{T}$ under diurnal conditions. The inertial subrange scale ends are approximately $0.3 \mathrm{~Hz}$ and $7.0 \mathrm{~Hz}$ [4]. These values have been calculated through using the method based on isotropy coefficient calculus using the Haar wavelet transform [23].

\section{RESULTS AND DISCUSSION}

As mentioned earlier, the absence of interaction between the CS dynamics in different scales implies to have the quantity $\rho_{w, \Delta w}$ equals to zero. The mean values and standard deviation bars for $\rho_{w, \Delta w}$ as a function of increment in $r$, converted to time using the Taylor's hypothesis are shown in Fig.3. These values correspond to the vertical wind velocity for both the periods of the day. We note that $\rho_{w, \Delta w}$ have a maximum value of approximately 34.1 seconds, obtained for both periods. Note that this value is out of the inertial subrange obtained $(0.15$ to 3.33 seconds, approximately). Thus, we observe that there exists interactions between large scales eddies present in the velocity field.

In addition, another important scaling aspect is that we compare the results between the diurnal and nocturnal periods. The $\rho_{w, \Delta w}$ for the nocturnal period presents superior values for time interval less than 17 seconds and smaller values for time interval between 17 to 136 seconds, in comparison to the diurnal period. The differences found between both periods may be an indicative of the role of the thermal gradient 



FIG. 1: Dyadic scales obtained from vertical wind velocity using the Haar wavelet transform. The top panel is the original time-series and, the bottom panel is the sum of the scales between 8 to 13 scales.

over Amazonian forest, because the forest canopy is an important absorbing mechanism of heat during the day and emitter of heat during the night. This is an important result, showing the influence of the scaling stratification on the great scales of vertical movements.

The difference between diurnal and nocturnal period found above reveal the importance of the thermal effects on the Amazonian physical environment. Into the surface boundary layer exist structures like ramps that are associated with vertical thermal gradients of the atmosphere [24]. According to this interpretation, it is possible that these structures, created by thermal effects, promoting convective movements, are the underlying process responsible for the higher correlation between the vertical wind velocity and temperature, explaining the value of 34 seconds found in Fig.3. These CS like ramps would be very weak in the nocturnal period, justifying the lower values found of $\rho_{w, \Delta w}$ during this period when compared to the diurnal one.

To better investigate the aspect mentioned above, we performed the Cross Wavelet Power (XWP) applied to time- 


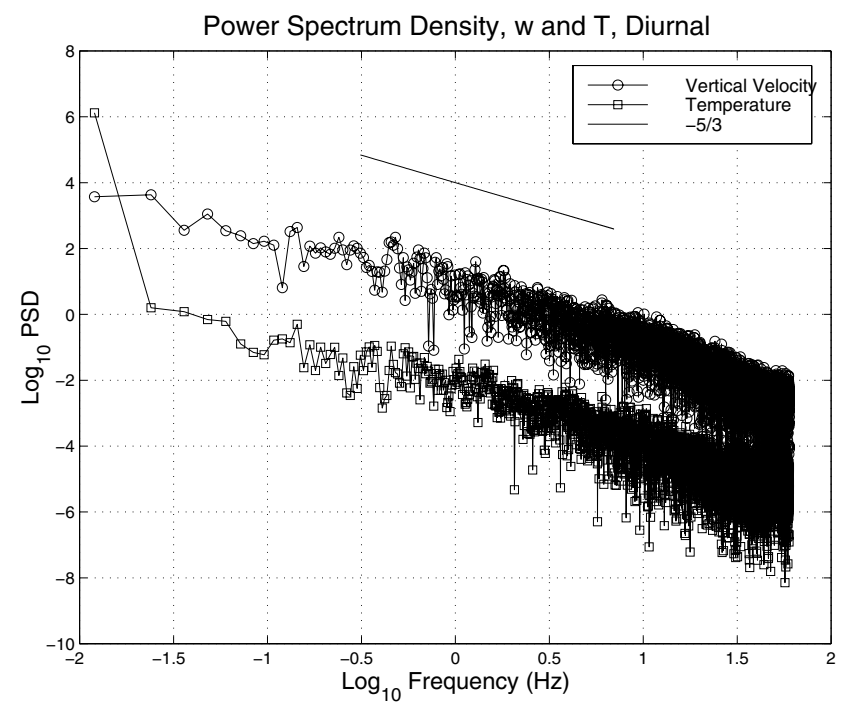

FIG. 2: Power Spectrum Density (PSD) for the vertical wind velocity and temperature measured in Rebio Jaru (Amazonia), diurnal period. The line represents the $-5 / 3$ declivity. The PSD for vertical wind velocity was shifted to up for better visualization.

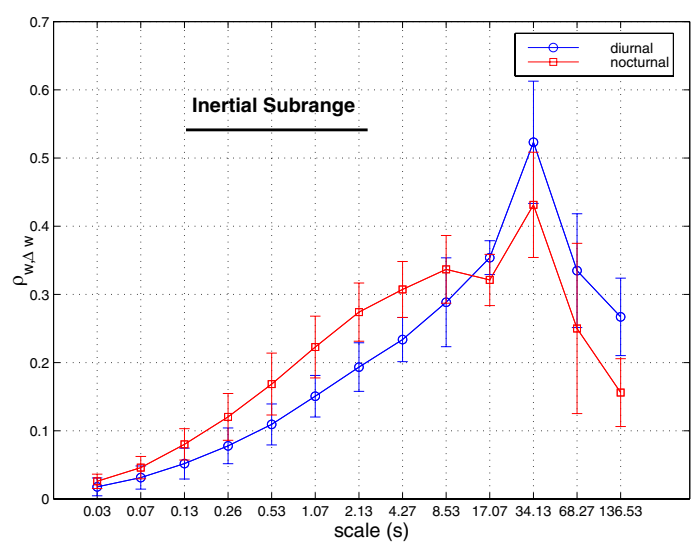

FIG. 3: Correlation Coefficient for the vertical wind velocity measured in Rebio Jaru (Amazonia), diurnal and nocturnal periods. The time axis is in $\log$ coordinates.

series of temperature and vertical wind velocity, measured at same time during both periods. Figs.4a and $4 \mathrm{~b}$ show the time-series of vertical wind velocity and temperature, respectively, for the diurnal period. Fig.4c shows only one example of the XWP for vertical wind velocity and temperature, at 12:00 hours on 068 Julian day of 1999. We observe that the correlation between both the variables increase just in the period where there is the presence of the CS like ramp, shown in Fig.4b, corresponding of the time interval between approximately 1.7 to 3.7 minutes. This interval corresponds to 2 minutes or 120 seconds of CS life-time as shown in Fig.4b.

We performed the Global Wavelet Spectrum (GWS) to study the dominant periods of the XWP for the diurnal and nocturnal conditions. The results are shown in Fig.5. For the diurnal period, we observe in Fig.5 (upper panel), three relative maximum in cross-wavelet ( 42,65 and 100 seconds). It is interesting to note that the relative maximum of 100 seconds may be associated with the CS like ramps showed in Fig.4b. For the nocturnal period, the same behavior is observed in Fig.5 (lower panel), from where it is possible to find three relative maximum in the cross-wavelet: 25,38 and 84 seconds.

In order to compare the results, we also performed the Fourier co-spectrum between $w$ and $T$ for both periods. An important difference between the two procedures is that, the GWS for XWP gives us the contribution of each periods to cross-correlation, whereas the co-spectrum gives us the contribution of each periods to energy. Fig.6 shows the results for the co-spectrum. We do not use loglog plot for a better comparison. In the first analysis, we note the difference between both periods, showing the influence of stratification in the vertical fluxes. Bolzan [4], have reported the non-extensive parameter for temperature, $q_{T}$, for above and below the top of same forest canopy in Amazonia, where this parameter is a direct measurement of intermittency. They showed that, under both the diurnal and nocturnal conditions, the parameter $q_{T}$ has higher values above the canopy and that, this could be attributed to the existence of large-scale like-ramp coherent structures in temperature fields, above and also below forest canopy. We recall Chen and collaborators [25] that these structures are responsible for the most of the sensible heat transport downwards the top of canopy.

In the second analysis, we observe the common periods present in both day and night time, as 37 and 133 seconds. Some differences in the values were found in GWS but are within error limits. The common period of 37 seconds agrees with the value obtained through $\rho_{w, \Delta w}$.

¿From the results obtained here, we can conjecture the following physical picture: The simple fact that the Amazonian forest has roughness in its transition surface (from the vertical forest to the outside environment), that is an important condition to create turbulent eddies in vertical velocity fields, is also important to characterize the thermal properties of the transition surface. During the day, the Amazonian forest stores the heat from short-wave infrared incoming there, creating differences of temperature in upper air layers. This promotes a strong vertical thermal gradient, creating CS like ramps to decrease the strong gradient between the warm air layer just above the forest and layers above it. This fact was mentioned as important factor in studies of third-order statistics ([7] and references there in). These CS, with life time of 120 seconds promote the increase of vertical velocity during the day. This fact shows an increment of the vertical wind velocity correlation coefficient in the scale of 34 seconds. Recent results by Cava [26], show that the presence of CS like ramps occur frequently during clear sky nights, and their time scales are about 30-60 seconds. In our results, we observe CS like ramps in almost all time-series of temperature during the day and, for the night period, it is possible that the CS like ramps would not exist or be less active, showing inferior values of the $\rho_{w, \Delta w}$, when compared to the diurnal period. Furthermore, it is important to note that, the results by Cava [26] were obtained over a uniformly planted loblolly pine forest and, thus, it is 

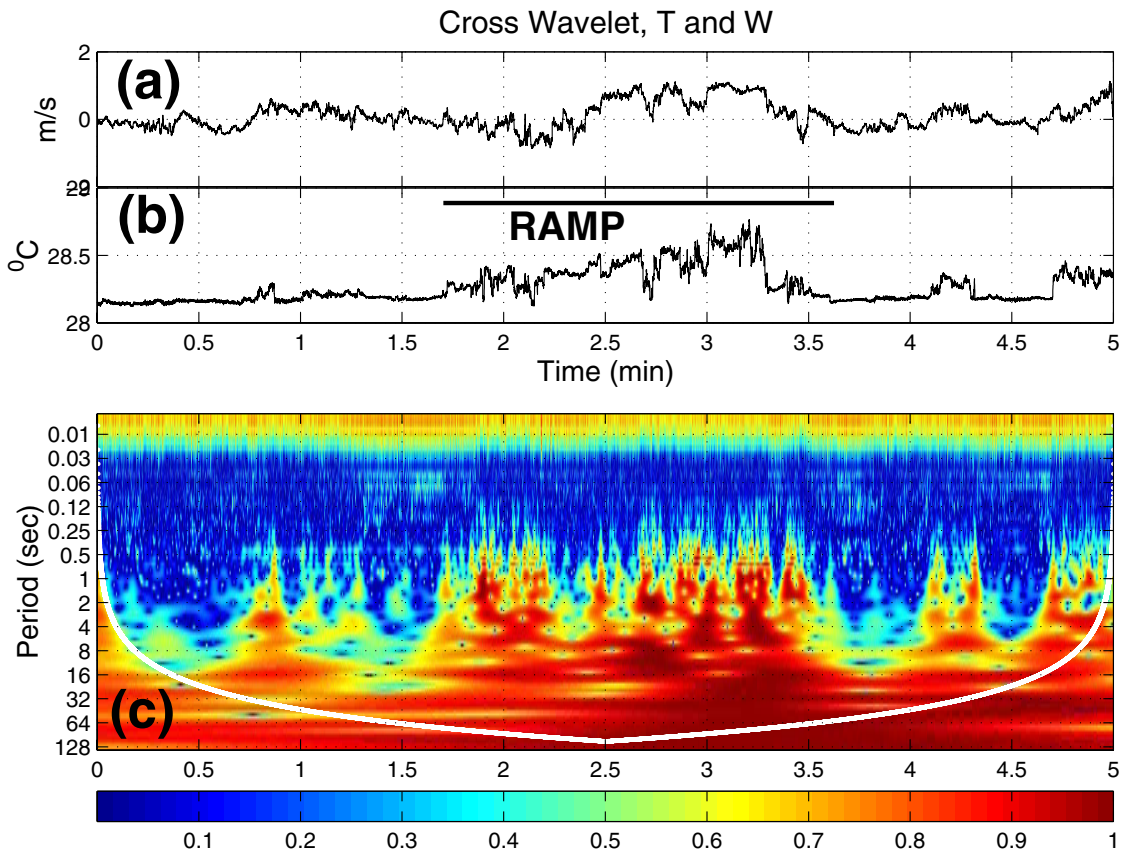

FIG. 4: (a) Five minutes of the Vertical wind velocity; (b) Temperature time-series, measured in same time at 12:00 hours, 068 julian day; (c) Cross Wavelet Transform applied in temperature and vertical wind velocity time-series. The white line is the influence cone.
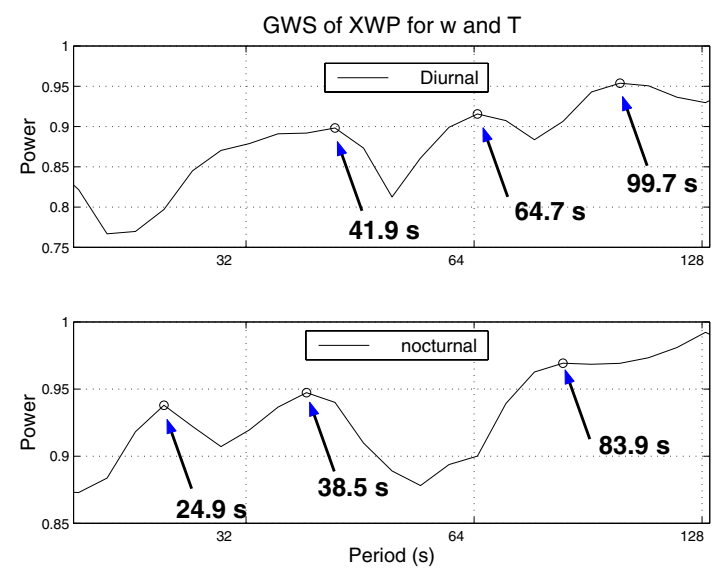

FIG. 5: Loglog plot of the $\mathrm{W}$ and $\mathrm{T}$ time-series for diurnal and nocturnal period of the XWP.

very different compared to the rain forest as Amazonia. This aspect would be a sufficient condition for modifying all turbulent structures, since the Amazonia forest is a more dense vegetation and, thus, it has higher capacity of heat storage during the day, creating the vertical thermal gradient, as mentioned earlier. However, these results show that there interactions exist between variables vertical wind velocity and temperature and, also, these interactions depends on the atmospheric stability conditions.

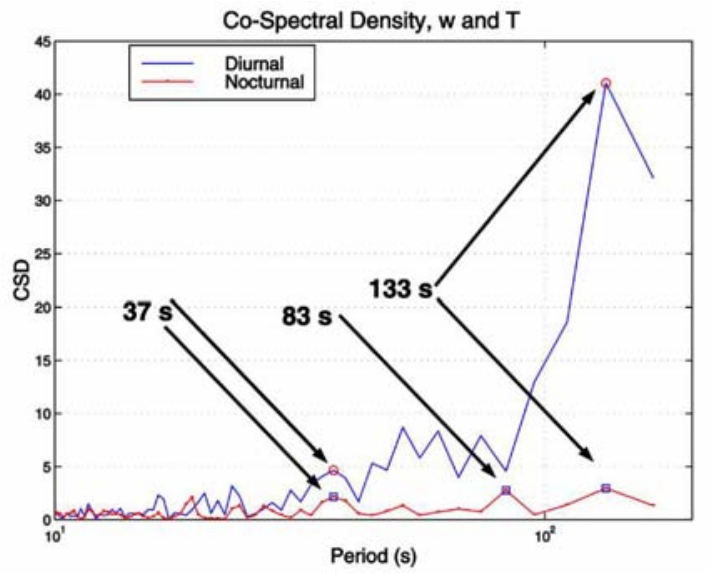

FIG. 6: Loglog plot of the $\mathrm{W}$ and $\mathrm{T}$ time-series for diurnal period.

\section{CONCLUSION}

We have analysed the turbulent time-series of the vertical wind velocity and temperature, measured in Amazonian forest, during the wet season Campaign of LBA (Large Scale Biosphere Atmosphere Experiment in Amazonia) in 1999 year, and sampled at $60 \mathrm{~Hz}$. The data were used to investigate the influence of coherent structures like ramps from temperature time-series in the eddies of large scales in wind velocity time-series, both measured over Amazonian forest in the diurnal and nocturnal periods. 
Using the methodology proposed by Katul [8], we observed the maximum correlation in 34 seconds for the vertical wind velocity for both the nocturnal and diurnal conditions. Some correlation differences between nocturnal and diurnal conditions were observed. The XWP shows that this difference is associated with thermal CS, like ramps, present in the temperature time-series. This fact was also corroborated by the co-spectrum analysis between vertical wind velocity and temperature.

The agreement between the results obtained from the procedures used in this work, can be summarized as follow:

a) The Influence of scaling stratification comes as a result from all procedures used in this work. In the Amazonia forest, due to dense vegetation, thermal effects dominate the turbulent structure against mechanical effects during the day. This result shows the importance of the thermal effects in the Ama- zonian forest;

b) Maximum correlation coefficient $\left(\rho_{w, \Delta w}\right)$ of the vertical wind velocity is 34 seconds, for both periods;

c) The value of 34 seconds is associated with the presence of the CS ramps present in the temperature time-series. Thus, these CS ramps are an important source of the correlation between the vertical wind velocity and temperature, as shown by XWP, GWS and co-spectrum of $w$ and $T$.

\section{ACKNOWLEDGEMENTS}

We thank the finnantial support from CAPES and the referee for valuable suggestions and comments. The M. J. A. Bolzan was supported by FAPESP (grant n. 2005/00511-9).
[1] J. C. R. Hunt, Eddy dynamics and kinematics of convective turbulence. In: Buoyant Convection in Geophysical Flows. Plate, E. J. et al. (Eds), Kluwer Academic Publishers, Netherlands, 41-82, 1998.

[2] F. M. Ramos, R. R. Rosa, C. Rodrigues Neto, M. J. A. Bolzan, and L. D. A. Sa, Nonlinear Analysis-Theory Methods \& Applications 47, 5 (2001).

[3] I. N. Esau, T. J. Lyons, Agricultural and Forest Meteorology 114, 3 (2002).

[4] M. J. A. Bolzan, F. M. Ramos, L. D. A. Sá, C. Rodrigues Neto, and R. R. Rosa, Journal of Geophysical Research 107, D20, doi:10.1029/2001JD000378, (2002).

[5] S. Toh, T. Itano, Journal of Fluid Mechanics 524, 249 (2005).

[6] M. J. A. Bolzan, Y. Sahai, P. R. Fagundes, R. R. Rosa, F. M. Ramos, and J. R. Abalde, Journal of Atmospheric and SolarTerrestrial Physics 67, 14 (2005).

[7] Z. Warhaft, Annual Review of Fluid Mechanics 32, 203 (2000).

[8] G. G. Katul, M. B. Parlange, J. D. Albertson, and C. R. Chu, Boundary-Layer Meteorology 72, 1-2 (1995).

[9] S. K. Robinson, Annual Review of Fluid Mechanics 23, 601 (1991).

[10] A. C. L. Chian, E. L. Rempel, E. E. Macau, R. R. Rosa, and F. Christiansen, Physical Review E 65, 3, 035203 (2002).

[11] S. Oughton , W. H. Matthaeus, Nonlinear Processes in Geophysics 12, 3 (2005).

[12] M. J. A. Bolzan, Braz. J. Phys. 35, 3 (2005).

[13] K. R. Sreenivasan, Physics of Fluids 8, 189 (1996).

[14] L. Mydlarski, Z. Warhaft, Journal of Fluid Mechanics, 358, 331 (1998).
[15] D. R. Osborne, J. C. Vassilicos, and J. D. Haigh, Physics of Fluids 17 (2005)

[16] F. M. Ramos, M. J. A. Bolzan, R. R. Rosa, and L. D. A. Sá, Physica D 193, 278 (2004).

[17] C. Torrence, G. P. Compo, Bulletinf of the American Meteorological Society 79, 61 (1998).

[18] A. Grinsted, J. C. Moore, and S. Jevrejeva, Nonlinear Processes in Geophysics 11, 561 (2004).

[19] M. J. A. Bolzan, Revista Brasileira de Ensino de Física 26, 1 (2004).

[20] C. Rodrigues Neto, A. Zanandrea, F. M. Ramos, R. R. Rosa, M. J. A. Bolzan, and L. D. A. Sá, Physica A 295, 215 (2001).

[21] G.-M. Le, J.-L. Wang, Chinese Journal of Astronomy and Astrophysics 3, 5 (2003).

[22] M. A. F. da Silva Dias, S. Rutledge, P. Kabat, P. Silva Dias, C. Nobre, G. Fisch, H. Dolman, E. Zipser, M. Garstang, A. Manzi, J. Fuentes, H. Rocha, J. Marengo, A. Plana-Fattori, L.D.A. Sá, R. Alvalá, M. Andreae, P. Artaxo, R. Gielow, and L. Gatti, Journal of Geophysical Research 107, D20 (2002).

[23] J. R. Kulkarni, L. K. Sadani, and B. S. Murthy, Boundary Layer Meteorology 90, 217 (1999).

[24] K. T. Paw U, Y. Brunet, S. Collineau, R. H. Shaw, T. Maitani, J. Qiu, and L. Hipps, Agricultural and Forest Meteorology 61, 1-2 (1992).

[25] W. Chen, M. D. Novak, and A. Black, Boundary Layer Meteorology 84, 99 (1997).

[26] D. Cava, U. Giostra, M. Siqueira, and G. Katul, BoundaryLayer Meteorology 112, 129 (2004). 